\title{
Libraries and Networks: An Irresistible Combination
}

\author{
by Alan R. Blatecky
}

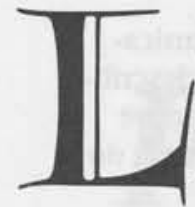

ibraries and networking are approaching a watershed that will radically change the way we think, the way we get information, and the way we approach education, research, and business. For the last couple of decades, we have been dealing with technologies that enable us to do things faster and quicker.

Computing power continues to scale to the point where yesterday's supercomputers are today's advanced workstations. Memory, storage and networking have also scaled; instead of Kbytes (thousand bytes) of memory, we talk of Mbytes (million bytes); instead of Mbytes of storage we talk of Gbytes (giga, a billion bytes) and Tbytes (a trillion); instead of megabit networks, we talk of gigabits. ${ }^{1}$

In addition, there is already a great deal of effort on the next generation of capabilities that are several orders of magnitude more powerful still - 256 megabyte RAMs, Pbytes (1000 trillion bytes) of storage, Teraflops (trillion floating point operations per second) of compute power, and Terabit (trillion bits per second) networks .

Libraries will not be a major force in the developing information age if they do not aggressively adopt and adapt technology to meet the information needs of their constituents.

\section{Information growth}

- In the information arena, we see similar statistics.

- Three new accessible databases appear daily (more than five thousand currently). - The world's amount of information doubles every five years.

- More facts and information became available in the last thirty years than in the previous five thousand years. ${ }^{2}$

- One day of The New York Times has more information than a lifetime in seventeenth century England. 3

- Ninety percent of all information published since 1979 is/was digital.

- Fifty-six thousand new books are published each year in the US alone (worldwide the number is in excess of two hundred fifty thousand). 4

- More than one million magazine articles are published yearly. 5

- Forty thousand scientific articles are published each year (one every thirty seconds). - Eight hundred new periodicals are published per year (some only digital, some with video). 6

This means that top libraries must double in size every twelve to fourteen years just to accommodate the growth in information, to say nothing of expansion of information or libraries. ${ }^{7}$

\section{Cost trends}

While the unit costs of technology continue to decrease dramatically each year, library construction costs are increasing. The capital cost to accommodate one hundred volumes is approximately two hundred and fifty dollars. ${ }^{8}$ This does not include furnishings, operational costs (HVAC, maintenance), or staffing. On the other hand, the costs of electronic mass storage, compute power, and networks continue to decrease.

For example, the cost per megabyte of storage has dramatically decreased over the last decade.

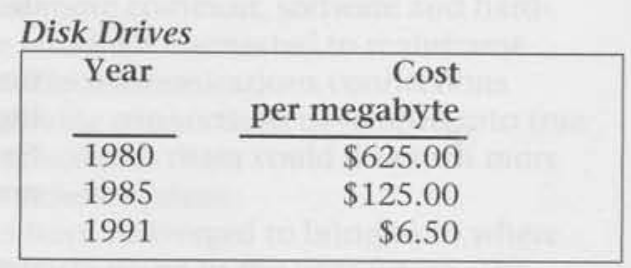

By 1995 the cost per megabyte of storage is likely to be a tenth of today's cost, or around sixty cents per megabyte. However, there are alternative types of electronic storage which are much more cost effective than hard disk drives. The advances in optical and dense magnetic tapes provide per unit costs that are several orders of magnitude less expensive.

\begin{tabular}{|lr|}
\hline \multicolumn{2}{|c|}{ Mass Storage Costs (1990) } \\
Rewritable Optical & $\$ 0.39$ \\
CD-ROM & $\$ 0.008$ \\
Digital Recorder & $\$ 0.001$ \\
Magnetic Cartridge & $\$ 0.05$ \\
Optical Tape & $\$ 0.005$ \\
8mm Helical Tape & $\$ 0.005$ \\
\hline
\end{tabular}

While individual workstation costs continue to decrease each year, the more important factor is the significant accompanying increase in workstation power. Mainframe power of a few years ago is available now at the desktop; an IBM RS6000 workstation now has the power of a single processor CRAY X-MP supercomputer. Workstation displays have benefited greatly from the R\&D advances increasing screen resolution and quality. High resolution screens are available now at a fraction of the cost of five years ago, and this trend will continue at an even more rapid rate as high definition television and graphics systems are developed.

\section{Network increases}

Similar cost reductions and performance increases are also taking place in networking. Where dial-up modems operating at three hundred and twelve hundred bps (bits per second) once were standard issue, 
modems approaching thirty-four thousand bps in speed are now available. Network backbone link speeds of 9.6 and $19.2 \mathrm{Kbs}$ (kilobits per second, e.g., 9.6 Kbs $=9,600$ bits per second) are quickly being replaced by individual network speeds of $56 \mathrm{Kbs}$ and T1 (1,544,000 bps) lines. T3 lines ( $45 \mathrm{Mbs})$ and FDDI (100 Mbs) networks are beginning to be implemented all across the country. Recent passage of the High Performance Communications and Computing (HPCC) Act of 1991 will help ensure the

deployment of high-speed networks (gigabit and beyond) across the nation.

However, as network speeds move to $\mathrm{T} 3$ and on to gigabits, faster is no longer an adequate way to describe what is happening. Whereas a gigabit network is one thousand times faster than a megabit network, the real change is one of scope and capability. Gigabit speeds provide the capability to do things differently. The primary mode of operation is no longer limited to text (i.e., characters and sym-

\section{Text and bits}

To help bridge the gap between printed text and electronic storage, it is necessary to understand how text and images translate into bits.

\section{Text versus Storage}

Document Number of bits

1 page of text

10 pages of text

1000 pages of text

25 page article with 10 color images

Library of Congress holdings

abbr.

$250,000250 \mathrm{Kbs}$

$25,000,000 \quad 25 \mathrm{Mbs}$

$900,000,000900 \mathrm{Mbs}$

200 trillion 25 TBs-2 PBs (1,000 TBs)

Another way of looking at the information is to translate storage into document size;

Amount of storage abbr.

\begin{tabular}{ll}
\hline $1,000 \mathrm{KBS}$ & $1 \mathrm{MBS}$ \\
$1,000 \mathrm{MBS}$ & $1 \mathrm{GBS}$ \\
$1,000 \mathrm{GBS}$ & $1 \mathrm{TBS}$ \\
$1,000 \mathrm{TBS}$ & $1 \mathrm{PBS}$ \\
ASCII Text & $(1 \mathrm{TBS})$
\end{tabular}

\section{Storage versus documents}

Number of documents

4 high resolution images or 320 pages of text

4,000 images or 320,000 pages of text

4 million images or 320 million pages

4 billion images or 320 billion pages

$(42,500$ trees $)$

Translating even further, the capabilities already exist in current technology to make electronic storage compellingly attractive simply in terms of cost alone. A single $19 \mathrm{~mm}$ tape can hold $25 \mathrm{~Gb}$, or more than 1,300 medium-sized books. Based on equivalent floor space, electronic storage can accommodate more than fifteen hundred times as many books and articles as does storage of the physical documents themselves.

An analysis of CD-ROM shows even larger gains. But, this overlooks perhaps the most significant values of electronic storage - remote access and shared resources. People who have access to a network that includes libraries have access to those resources without having to travel to the library to see the physical documents. The information is available to them twenty-four hours a day at their place of work, study, or home.

This same type of table can be constructed for transmission speeds.

\section{Transmission times}

\begin{tabular}{l|rrrr}
\multicolumn{5}{c}{ Network Speeds } \\
\hline Document & $9.6 \mathrm{Kbs}$ & 1.5 & $\mathrm{Mbs}$ & $1 \mathrm{Gbs}$ \\
\hline 1 page & $2.6 \mathrm{sec}$, & $.02 \mathrm{sec}$ & $.00003 \mathrm{sec}$ \\
100 pages & $4.5 \mathrm{~min}$ & 1.7 & $\mathrm{sec}$ & $.003 \mathrm{sec}$ \\
25 page article with 10 images & $28 \mathrm{hrs}$ & 10.8 & $\mathrm{~min}$ & $.9 \mathrm{sec}$ \\
$\begin{array}{l}\text { Library of Congress } \\
\quad \text { lower limit of 25 TBS }\end{array}$ & 661 yrs & 1,543 days & $56 \mathrm{hrs}$ \\
$\quad$ upper limit of 2 PBs & 52,880 yrs & 338 yrs & 186 days \\
\hline
\end{tabular}

These tables clearly illustrate the new world for information and libraries. Documents and articles can be accessed and retrieved in seconds at gigabit speeds. This is in sharp contrast to the networks and technologies that libraries typically use today ( 9.6 to $56 \mathrm{Kbs}$ link speeds), where it can take hours to electronically retrieve a single document or article (books could take days). The result is that only selected documents are made available - usually those not containing large sets of data or images. bols), but relies on visual information; images will dominate the way we use networks and interact with information.

The increases in network speeds and computer power as well as reduction in storage costs have tremendous implications for libraries and information. If technology can provide adequate digital storage, transmission and display of images in real time, then digital libraries are no longer relegated to being future images or ideals. Digital libraries themselves become the basis for library development and deployment. From this point on, it will be very difficult to justify physical library expansions based primarily on volumes and print.

\section{New paradigms}

At the same time, it is clear also that libraries must be prepared to do things differently. Libraries will not be a major force in the developing information age if they do not aggressively adopt and adapt technology to meet the information needs of their constituents. For example, the normal operating procedures of electronic document delivery associated with slowspeed networks and technology are totally inappropriate for high-speed networks. It is not adequate simply to have the card catalog online. The goal has to be to have the full text online so it can be retrieved and "browsed" over the network just as the patron would do when walking down an aisle of books and journals.

It is also important to note that the definition of information is facts, figures and images. One way to help illustrate this is the four tier model:

\section{Raw Data \\ Information \\ Knowledge \\ Wisdom}

Information is becoming much more interactive; users find it essential to readily navigate between raw data and information in real time in order to get the knowledge and understanding they need. This is an iterative process which becomes ever more important as the amount of available information continues to increase (e.g., how can the user quickly sift through the data to get at crucial information). In other cases, the process itself becomes part of the growing database. For example, an educator reviewing results of a survey on teaching styles would be able to add his/her experience. The result is that the database grows every time it is used; that is, the new experience is incorporated into the database.

These types of applications are already taking place through computer conferencing and promises to become a significant source of information and ex- 
pertise. This points out that libraries must begin seriously to adopt several paradigm shifts in order to usher in and develop the next-generation library and information center. One of the shifts is that libraries must rapidly respond to accommodate these "new" types of information sources. Another involves navigation. On the one navigation expertise to assist users. On the other hand, in many cases, librarians will hand, libraries need to develop electronic

standards for interoperability and resource sharing. While these are significant factors that involve not only economics, politics, and inertia (substantial investments and infrastructures used to doing business as they always have), the marketplace and user needs will drive the required changes over time. The question is not if these issues will be resolved, but when; and the more pertinent question is who is going to take leadership to provide the information for the electronic world. The discussion has also deliberately side-stepped many issues dealing with electronic information as these are beyond the scope of this article.

In essence, the tremendous changes in networking, communications, and computing mean that geography and time will no longer be obstacles. Where you are located will have little impact on what you can do or learn. Resources, expertise, and innot be intermediaries, as the users will access the information directly.

The discussion so far has centered on hard copy and text. However, there are many other rapidly developing types of electronic information such as databases, electronic journals, scientific visualization, and graphics. These assume that highperformance networks will be the primary interface for the vast majority of users. The growing importance of video, moving images, and high-resolution graphics to science, education, and business will require yet other new technologies and new approaches to handle what we call "information technology." Librarians will need to become conversant with a wide variety and ever-growing array of non-bibliographic forms of information.

These same users are not only "athome" in this electronic information world, but also are the vanguard for the future. These users depend on access to current information (discoveries and new ideas are shared within minutes) for their livelihood, and seek out those information repositories that have high-performance electronic capabilities. In many other cases, they can not wait until the information is available in print or travel to the source to get the materials. They want and need access to the video, images, and data within minutes or hours. (Interestingly, this sort of timeliness has contributed to the success of Cable Network News.)

The discussion has deliberately sidestepped many electronic information issues that are beyond the scope of this article. The topics range from copyright and publishing issues to those of technical seconds; from your office you will be able to provide yet another capability with face-to-face communications among offices, libraries, and so on. It will greatly enhance collaboration and interaction between users and information providers.

\section{Conclusion}

In conclusion, a number of factors and opportunities face libraries that must be addressed if libraries are to be viable information centers for the next century:

- The willingness of librarians to adopt a different model of what it means to be a library - that is, a digital library. How does a library begin to position itself to make the transition to a digital library? Is it necessary that all libraries become digital, or to what extent?

- A major change in the organizational structure and culture of libraries. Libraries are organized in terms of supporting a central repository; the future model is going to be much more disbursed, requiring a flattening of the hierarchy and a move toward disbursed management of resources and staff.

- A move from increased holdings to increased access; the goal is to use electronic networks to reach remote physical resources.

- Libraries will have to adapt to using a wide range of classification schemes (access strategies) rather than try to fit all information into one classification system, as they draw upon information in interactive formats and from a wide range of non-library electronic (network) resources.

- Much more emphasis upon the sharing of resources among libraries from the outset. How can each library carve out a unique niche or set of holdings that will be of use to the much larger national or global community? It will require coordinated planning, purchasing and cataloging to avoid duplication.

- The acquisition of technical knowledge and expertise for all librarians. While the goal is not to require that librarians be computer programmers, it is essential that they be technically literate and have substantial technical resources (networking, storage and computing) in residence or on call. In addition, it is essential that the technology be integrated throughout the entire organization, from top to bottom.

Libraries and networks are approaching a watershed which will radically change the role of both. New paradigms are going to be required to effect the transitions. Only through a tight coupling of strategic information resources and expertise with ubiquitous, high speed network access, will we be able to increase our productivity, remain competitive, and sustain high quality education.

\section{References and Notes}

1 Bytes versus bits: 8 bits to every byte; bits indicated by lower case (Mbs), bytes by upper case (MBs).

2 Peter Large, The Micro Revolution Revisited (New Jersey: Towman \& Allanheld Company, 1984).

3 Richard Saul Wurman, Information Anxiety (Doubleday, New York, 1989).

4 Bowker Anmual: Library and Book Trade Almanac 36 th edition, compiled and edited by Filomina Simora, R.R. Bowker, 1991.

5 Steven Louis and Robert F. Rubeck, "Hypertext Publishing and the Revitalization of Knowledge," Academic Computing (May 1989): 22-32.

6 Science News 142, 5 (August 1, 1992): 78 .

7 Wilbur Schramm and William Porter, Men, Women, Messages, and Media; Understanding Human Communication (New York: Harper \& Row Publishers, 1982).

8 Wilson M. Stahl, "The Future Impact of High-performance Networks on Library Collection, Facilities, and Services," Metropolitan Universities 3, 1 (Summer 1992) in press. 